\title{
Análise sobre Sistemas baseados em Learning Analytics (LA) para apoiar as ações pedagógicas em Ambientes Virtuais de Aprendizagem (AVA) tipo Moodle
}

\author{
Anita Raquel da Silva - IFRS - anita@cinted.ufrgs.br \\ Marcelo Augusto Rauh Schmitt - IFRS - marcelo.schmitt@poa.ifrs.edu.br \\ Liane Margarida Rockenbach Tarouco - UFRGS - liane@penta.ufrgs.br
}

Resumo. O presente artigo é resultado da análise de sistemas baseados em Learning Analytics (LA) para apoiar as açõ es pedagó gicas dos docentes em cursos que utilizam Ambientes Virtuais de Aprendizagem (AVA). Realizou-se estudo bibliográfico para determinar que tipo de software vem sendo proposto por pesquisadores para apoiar as atividades docentes em cursos a distância de graduação e pós-graduação. Pretende-se verificar os trabalhos realizados que forneçam subsídios diretamente para os professores a fim de que esses possam tomar decisõ es pedagó gicas. Exclui-se aqui sistemas de Learning Analytics (LA) que forneçam informaçõ es diretamente para os alunos e que apresentem características de predição automática. Ao final da análise constatou-se uma grande utilização de LA pelas instituiçõ es de ensino tendo como foco a entrega de informações para os tutores e docentes.

Palavras-chave: Learning Analytics, Relatório, Sistema, EAD, docente.

Analysis on Systems based on Learning Analytics (LA) to support pedagogical actions in Virtual Learning Environments (VLE) Moodle type

Abstract. This article is the result of the analysis of Learning Analytics (LA) based systems to support the pedagogical actions of teachers in courses that use Virtual Learning Environments (VLE). A bibliographic study was conducted to determine what type of software has been proposed by researchers to support teaching activities in undergraduate and graduate distance learning courses. It is intended to verify the works that provide subsidies directly to teachers so that they can make pedagogical decisions. This excludes Learning Analytics (LA) systems that provide information directly to students and have automatic prediction characteristics. At the end of the analysis it was found a great use of AT by the Education Institutions focusing on the delivery of information to tutors and teachers.

Keywords:Learning Analytics, Relatório, Sistema, EAD, teacher.

\section{Introdução}

O Learning Analytics (LA), está sendo amplamente utilizado dentro de contextos educacionais diversos, como ensino presencial, semipresencial e educação a distância (EAD). Devido a este novo panorama, procurou-se realizar um levantamento bibliográfico para verificar se a utilização de sistemas baseados em Learning Analytics dentro de um Ambiente Virtual de Aprendizagem (AVA) como o Moodle pode auxiliar o docente no acompanhamento do discente e, dessa forma, possibilitar mais disponibilidade de tempo ao docente com relação ao acesso às informaçõ es frequentemente geradas pelos discentes e armazenadas no ambiente. Com isso, poderá diminuir a sobrecarga advinda da coleta de informações necessárias para a elaboração de feedbacks e definir estratégias 
pedagó gicas mais personalizados para os discentes. Acredita-se que a utilização de recursos tecnoló gicos pelo docente de EAD seja o aspecto principal para a melhora do desempenho das suas atividades pedagógicas junto aos discentes.

A utilização de sistemas baseados em Learning Analytics (LA) dentro dos curso apoiados por Ambientes Virtuais de Aprendizagem (AVA) é relevante uma vez que contribuem no melhoramento do acompanhamento pedagó gico. Desta forma, o desenvolvimento de softwares que utilizam LA integrado a AVAs são relevantes já que tais sistemas entregam dados educacionais em formato de informaçõ es relevantes para auxiliar na melhoria do processo de ensino e aprendizagem do discente pelo docente.

Com o intuito de analisar as produções científicas relacionadas ao tema e verificar quais são as instituições que estão utilizando Learning Analytics aplicado à educação, a que tipo de curso está sendo aplicado, quais são os sujeitos que estão tendo acesso aos informaçõ es entregues, como estes softwares estão sendo desenvolvidos e quais são os ambientes onde está sendo aplicado, este trabalho tem como objetivo apresentar uma revisão bibliográfica de trabalhos nacionais e internacionais.

A seção seguinte apresenta informaçõ es relacionadas a um levantamento realizado com tutores de um curso de especialização a distância através de um questionário on line realizado. Os principais resultados, bem como as indagaçõ es apresentadas como resposta, serão analisados e discutidos.

\section{Trabalhos Relacionados}

Para considerar a inclusão dos artigos, foram eleitos alguns fatores que ajudaram a identificar a relevância dos estudos primários para responder ao objetivo desta pesquisa, alguns critérios de inclusão e exclusão foram criados. Os critérios têm origem em trabalhos correlacionados (NUNES, 2013); (ZIELINSKI; SCHMITT, 2015). Dessa forma, os critérios foram utilizados como parâmetros para auxiliar na seleção das publicações.

Os trabalhos pesquisados, principalmente dos últimos quatro anos, em sua grande maioria, apresentaram como foco principal o aluno e ferramentas de cunho educacional construídas especificamente para auxiliá-los na realização de suas atividades educacionais dentro dos ambientes de apoio a aprendizagem, através de envios de mensagens e indicaçõ es de materiais de apoio. Todavia, um sistema sem o suporte de um docente pode ofertar ao aluno apenas materiais que, através de parâmetros, foram mais clicados (acessados) por outros discentes, sem ter uma visão mais específica das reais necessidades daquele discente. Por esse motivo, acredita-se na importância das interações entre discentes e docentes na construção do processo de ensino e aprendizagem. Para que isto ocorra, é importante que o docente tenha um tempo adequado para realizar o acompanhamento pedagó gico junto aos discentes, sem ter que despender uma alta carga horária procurando e organizando informaçõ es a respeito do discente que estão dispersas no ambiente.

\section{Procedimentos Metodológicos}

Justifica-se que não foram considerados artigos publicados em 2018, uma vez que algumas bases de dados não divulgaram os trabalhos disponíveis em função do período em que a revisão foi realizada (primeiro semestre de 2018). A Tabela 1 apresenta as questões estabelecidas para a pesquisa. 
Tabela 1. Questões estabelecidas para a pesquisa

Item $\mid$ Pergunta

P1. Quem estáutilizando Learning Analytics?

P2. Onde está sendo utilizado sistemas de alerta e dashboard (relatórios)?

P3. Para qual público alvo está voltado?

P4. Qual Metodologia de desenvolvimento?

P5. Quais linguagens de programação foram utilizadas?

P6. Qual recurso utilizado?

P7. Está sendo utilizada em um AVA? Qual?

P8. Qual modalidade de ensino?

Fonte: Autora

Para ter uma visão mais geral do escopo deste trabalho realizou-se uma análise de trabalhos correlacionados em bases de pesquisa internacionais e nacionais, distribuídos entre as bases de pesquisas consultadas no intervalo de 2014 a 2017. As bases de pesquisa utilizadas foram:

- Informática na Educação: teoria \& prática

- Google Scholar

- Revista Novas Tecnologias na Educação (RENOTE)

- Scopus

- Simpósio Brasileiro de Informática na Educação (SBIE)

- Springer

- Congresso Internacional de Informática Educativa (TISE)

- Workshop de Informática na Escola (WIE)

Para examinar os bancos de dados selecionados, uma sequência para a pesquisa foi estabelecida tendo como base as perguntas desta pesquisa. Para tanto foi criada uma string composta por palavras-chave, para tanto foram consideradas as suas versõ es em inglês e português.

As strings utilizadas na pesquisa foram (report and "learning analytics"and "virtual learning enviroment"and "distance education") e (relató rio and "análise de aprendizagem"and "ambiente virtual de aprendizagem"and "educaçã o a distância"). Nesta sequência, o operador "AND" é usado para conectar dois termos.

Na Tabela 2 são apresentados os critérios de inclusão $(\mathrm{Cl})$ que foram definidos para uma primeira análise dos trabalhos:

\begin{tabular}{c|l}
\multicolumn{2}{c}{ Tabela 2. Critérios de Inclusão } \\
Item & Critério de inclusão \\
\hline $\mathrm{Cl}-1$ & Conter Learning Analytics \\
$\mathrm{Cl}-2$ & Conter Educação a Distância (EAD) \\
$\mathrm{Cl}-3$ & Conter relatório de acompanhamento \\
$\mathrm{Cl}-4$ & Conter docente \\
$\mathrm{Cl}-5$ & Ações pedagógicas \\
& \multicolumn{1}{c}{ Fonte: Autora }
\end{tabular}

O filtro realizado a partir dos três primeiros Critérios de Inclusão $(\mathrm{Cl})$ deu-se, principalmente, em relação a estrutura do sistema a ser desenvolvido para subsidiar as atividades de coleta e entrega de informações dos alunos aos docentes de cursos EAD. 
Os trabalhos que se enquadram em pelo menos um desses critérios foram considerados para serem analisados considerando os critérios de exclusão (CE). Os trabalhos que não se encaixaram em nenhum CE foram selecionados.

Os critérios de exclusão elencados para a análise dos trabalhos encontrado na pesquisa realizada através da string são os apresentados na Tabela 3.

\begin{tabular}{c|l}
\multicolumn{2}{c}{ Tabela 3. Critérios de Exclusão } \\
Item & Critério de exclusão \\
\hline CE-1 & Conter MOOC \\
CE-2 & Conter Educação semipresencial \\
CE-3 & Conter entrega de soluções \\
CE-4 & Conter o foco no discente \\
CE-5 & Conter o foco no tutor presencial \\
CE-6 & Ações automáticas ou automatizadas
\end{tabular}

Fonte: Autora

A partir da busca realizada pelas string informadas, foram encontrados 90 trabalhos relacionados com a temática do escopo deste trabalho. Destes, 30 foram descartados inicialmente por tratarem-se de capítulo de livro, dissertaçõ es, livros, relató rio, trabalhos de conclusão de curso e teses. Com a exclusão destes trabalhos, obtivemos como resultado 60 artigos, sendo apenas dois repetidos, finalizando com 58 artigos.

Foram aplicados aos artigos os critérios de inclusão e exclusão. Inicialmente foi realizada uma etapa de seleção, que consiste em ler os títulos, resumos e palavras-chave dos 58 artigos encontrados como resultado da busca pela string. Este processo foi realizado com a aplicação dos critérios de inclusão e exclusão, obtendo-se, no final desta fase, 13 artigos incluídos e 45 excluídos. É importante elucidar que cada artigo incluído se encaixou em todos os critérios de inclusão, visto que, para ser descartado, ele deveria encaixar-se em pelo menos um dos critérios de exclusão.

Em seguida, os 13 artigos selecionados foram lidos na íntegra, tendo como base para sua análise os critérios de inclusão e exclusão, a fim de definir quais contemplavam dentro de seu conteúdo os critérios. Por fim, restaram 7 artigos incluídos e 6 excluídos.

Os 7 artigos finais conseguiram responder parcialmente as perguntas estabelecidas para esta pesquisa, tendo cada um deles alguma dificuldade em apresentar respostas para todas as perguntas elaboradas. Por este motivo, acredita-se que a presente pesquisa se apresente como relevante.

No intuito de demonstrarmos a fragilidade dos sistemas atuais de entrega de informaçõ es sobre o discente dentro de ambientes como o Moodle, realizaremos a análise de alguns mó dulos pré-existentes no AVA citado e plugins que podem ser instalados como recursos para realizar o acompanhamento dos discentes. Para analisar ferramentas de suporte para o acompanhamento das açõ es dos discentes dentro de ambientes virtuais, selecionamos alguns dos relató rios de acompanhamento que fazem parte do Moodle e os examinamos a partir de suas disponibilização, organização e usabilidade.

\section{Análise dos resultados}

A análise será realizada tendo como base os 7 artigos encontrados como resultado da busca pelas string nas bases de pesquisa. 
Para tanto foram criadas duas representaçõ es visuais para mostrar a distinção dos trabalhos encontrados por repositó rios selecionados e dos artigos encontrados por ano em cada repositório. Na Figura 1 se pode observar que o maior número de publicações encontradas está concentrado no repositório Google Scholar ou como também é conhecido como Google Acadêmico, o que representa $63 \%$ de todos os trabalhos que foram descobertos. Os repositórios com menor número de publicações encontrada foram Informática na Educação: teoria \& prática e RENOTE com $2 \%$ cada.

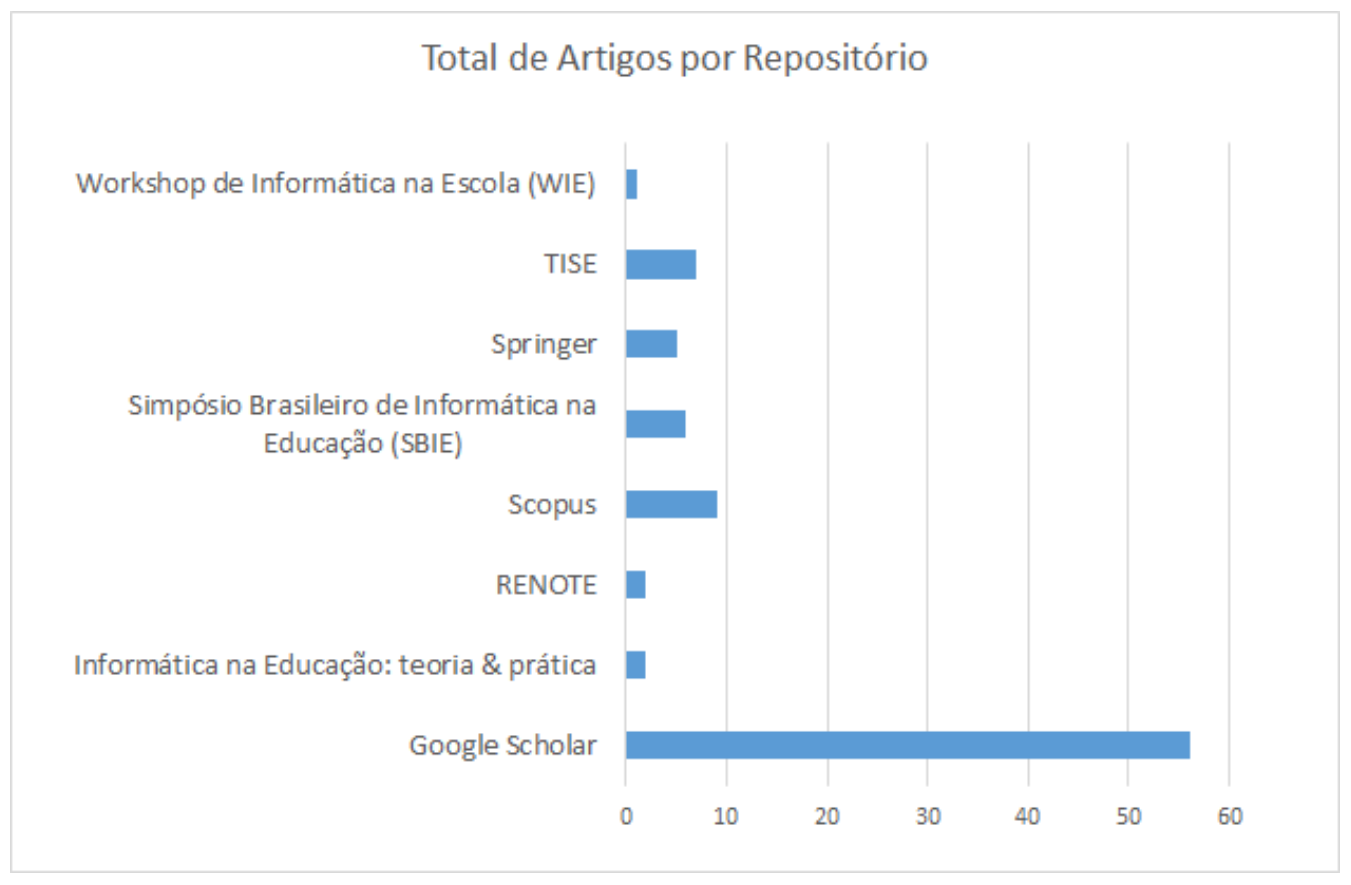

Figura 1. Gráficos relacionados aos artigos por repositório Fonte: Autora

A Figura 2 traz a representação gráfica exclusivamente dos artigos encontrados por ano nos repositó rios selecionados. Pode-se observar que o maior número de artigos publicados se encontra concentrados no ano de 2016 , o que representa $37,5 \%$ de todos os artigos que foram descobertos. Em contrapartida o ano com menor nú mero de artigos publicados foi 2014 , apresentando apenas $3,57 \%$, sendo que $58,62 \%$ destes artigos foram encontrados em repositó rios internacionais. Percebe-se que o nú mero de publicações aumentou nos últimos anos atingindo seu auge entre os três últimos anos referentes a esta pesquisa (2015 a 2017), possivelmente pelo fato de estarmos entrando na segunda fase do LA com a utilização de metodologia baseada exclusivamente na utilização de dados advindos de fontes digitais (eg AVAs e Redes Sociais). 


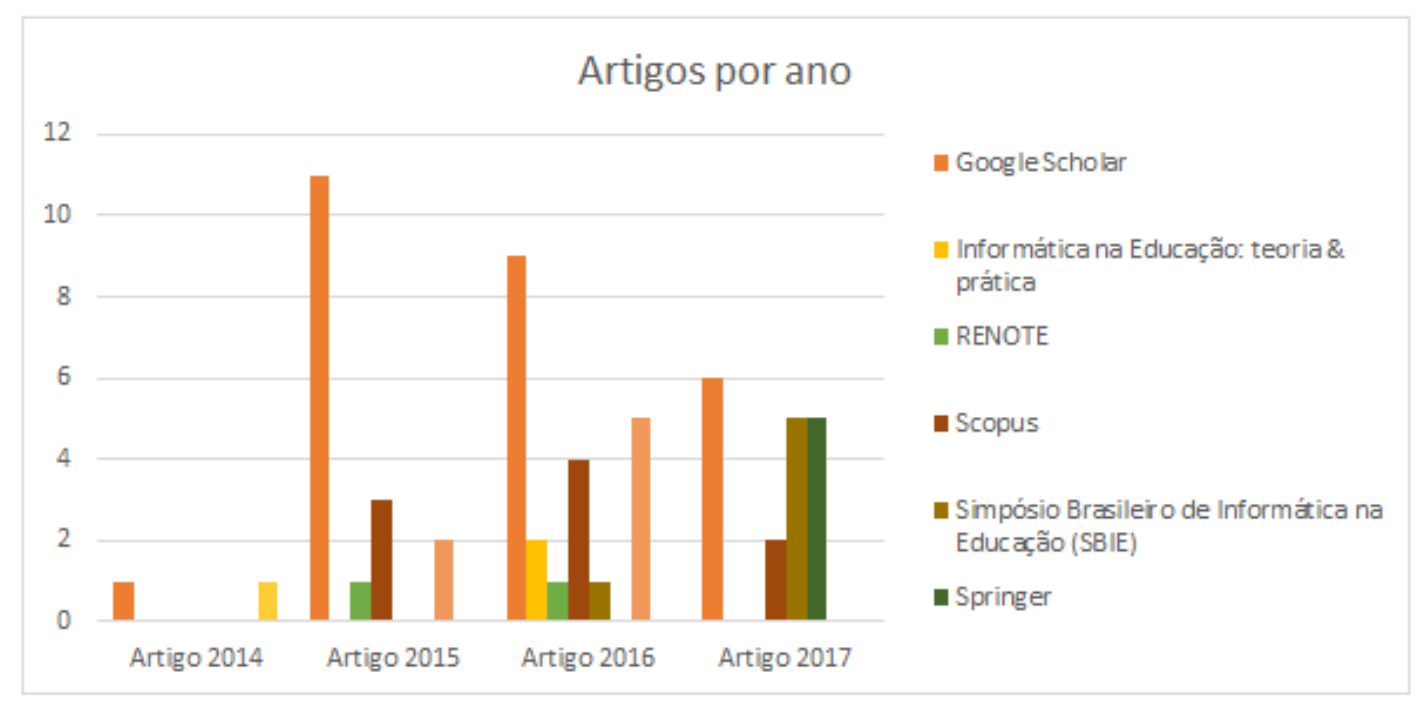

Figura 2. Gráficos relacionados aos trabalhos por ano Fonte: Autora

Considerou-se inicialmente que a maioria dos trabalhos realizados na área pesquisa seriam de origem externa ao Brasil, porem ao final da revisão encontrou-se um senário diferente, onde a maioria das pesquisas relacionadas foram feitas por pesquisadores de instituiçõ es nacionais. Com relação aos artigos considerados como relevantes à esta pesquisa sobre a utilização de sistemas baseados em Learning Analytics (LA) para apoiar os docentes dentro de AVA, apenas um apresentou sua pesquisa realizada fora do Brasil, sendo essa realizada na Universidade Aberta de Portugal em Lisboa. Os demais artigos $85,71 \%$ apresentaram pesquisas realizadas e aplicadas no Brasil. Destes sendo 50\% no Estado do Rio Grande do Sul - RS (Unisinos e IFRS em parceria com CINTED/UFRGS), do Amazonas - AM (Universidade Federal do Amazonas - UFAM), Minas Gerais - MG (Universidade Federal de Alfenas - UNIFAL) e Pernambuco - PE (UNIVASF). A Figura 3 apresenta a distribuição das pesquisas por países e regiões.

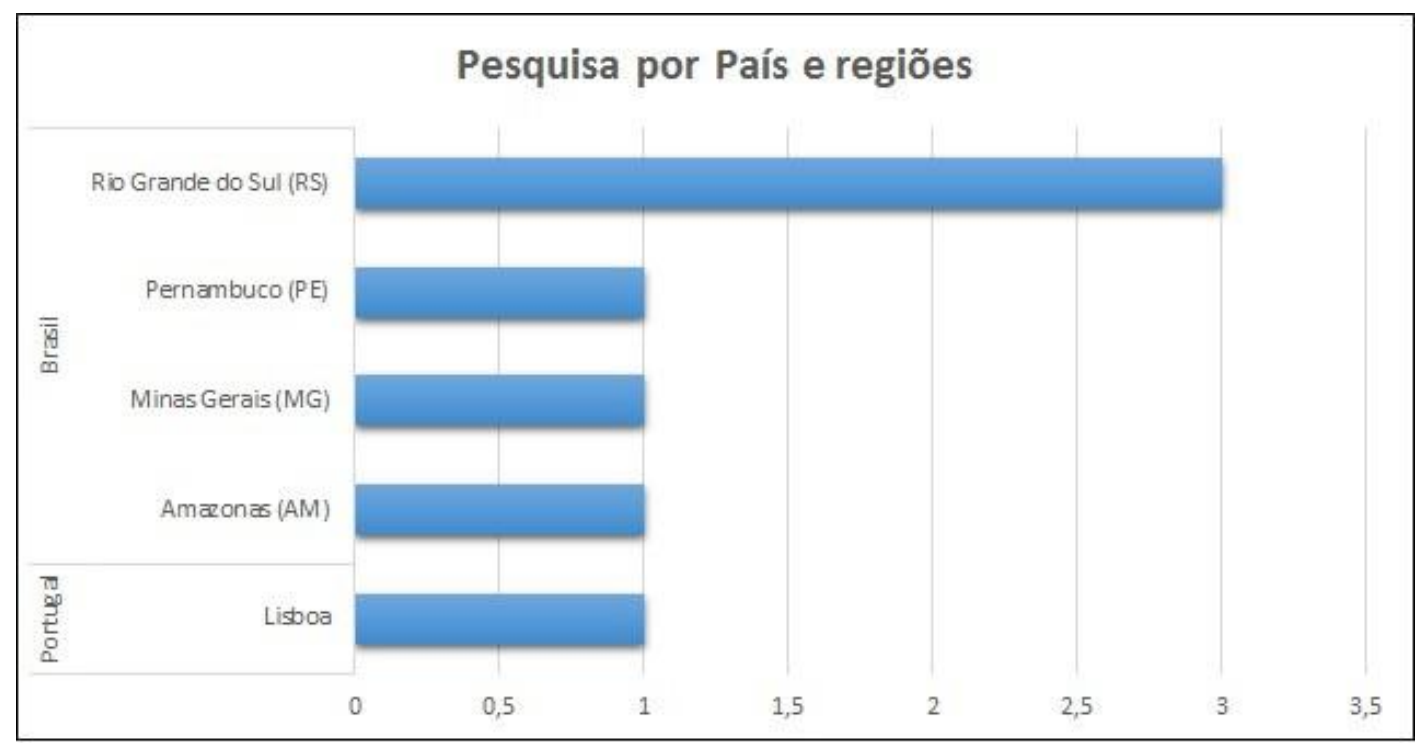

Figura 3. Pesquisas por País e Regiões

Fonte: Autora 
Quanto ao local das publicações dos artigos e características dos trabalhos, os dados apresentaram um maior nú mero de trabalhos foram publicados no Brasil e em Língua Portuguesa (5) com relação aos publicados Internacionalmente em Língua Inglesa (2). A maioria das publicaçõ es foram realizadas em Anais de congressos (4), as outras foram realizadas em Perió dicos (2) e Journal (1). Na Tabela 4 são apresentadas as informações relacionadas aos aspectos dos trabalhos.

Tabela 4. Aspectos dos trabalhos

\begin{tabular}{l|c} 
Tipo de publicações & $\%$ \\
\hline Língua Portuguesa & $71,43 \%$ \\
Língua Inglesa & $28,57 \%$ \\
Publicações Nacionais & $71,43 \%$ \\
Publicações Internacionais & $28,57 \%$ \\
Periódicos & $28,57 \%$ \\
Journal & $14,29 \%$ \\
Anais de congresso & $57,14 \%$ \\
\multicolumn{2}{c|}{ Fonte: Autora }
\end{tabular}

A pesquisa focou inicialmente na busca de trabalhos realizados em curso de especialização, principalmente no formato EAD, porém observou-se trabalhos realizados em vários níveis do ensino superior e a maioria delas realizadas em curso de graduação, ficando os cursos de especialização em seguida. No que concerne ao tipo de cursos onde as aplicações foram realizadas nos artigos investigados, estas aplicaçõ es foram encontradas principalmente e cursos superiores do tipo: Especialização (28,57\%); Extensão (14,29\%); Pós-graduação (14,29\%) e Graduação (42,87\%).

A pesquisa focou inicialmente na busca de trabalhos realizados em curso de especialização, principalmente no formato EAD, porém observou-se trabalhos realizados em vários níveis do ensino superior e a maioria delas realizadas em curso de graduação, ficando os cursos de especialização em seguida. No que concerne ao tipo de cursos onde as aplicaçõ es foram realizadas nos artigos investigados, estas aplicaçõ es foram encontradas principalmente e cursos superiores do tipo: Especialização (28,57\%); Extensão (14,29\%); Pós-graduação (14,29\%) e Graduação (42,87\%).

Com relação ao tipo de sujeito que está sendo beneficiado com a utilização de metodologias de Learning Analytics, procurou-se verificar quantas pesquisas estavam focando em auxiliar o trabalho dos tutores junto aos alunos dos cursos de ensino superior. Foi satisfató rio verificar que mais de cinquenta por cento dos trabalhos considerados como relevante para esta pesquisa apresentou como sujeito principal os tutores e em seguida os professores. No que se refere aos tipos de sujeitos envolvidos nas pesquisas realizadas, os artigos apresentaram quatro grupos específicos: Tutores, Professores, Gestores e Alunos.

Para representar os sujeitos que foram foco pelas pesquisas realizadas nos artigos considerados criou-se a Tabela 5, de forma a apresentar os tipos de sujeitos indicados nos artigos de forma individual como em seus respectivos grupos pesquisados.

Tendo em vista a proposta com relação a utilização da metodologia de tratamento dos dados, 71,43\% artigos informaram ter utilizado metodologia de Learning Analytics (LA), porém não identificaram os passos utilizados para tal. Com relação aos demais artigos foram obtidos o percentual de 14,29\% em relação Education Data Mind (EDM) e $14,29 \%$ para Desing Base Research (DBR). 
Tabela 5. Grupos de sujeitos

\begin{tabular}{l|c} 
Sujeitos/Grupos & $\%$ \\
\hline Tutores & $57,14 \%$ \\
Professores & $42,86 \%$ \\
Gestores & $28,57 \%$ \\
Alunos & $14,29 \%$ \\
Tutores e Gestores & $14,29 \%$ \\
Docentes e tutores & $14,29 \%$ \\
Professores e alunos & $14,29 \%$ \\
\multicolumn{2}{c}{ Fonte: Autora }
\end{tabular}

Com relação as linguagens de programação utilizadas foram obtidas as seguintes: WEB (PHP - Javascript); ASP.NET C\#, SQL SBd, OWL; WEB (PHP - Javascript); PHP, Jquery, highCharts.js, HTML e CSS; Java; A4Learning framework; contudo não foi possível identificar em um dos trabalhos se foi utilizado linguagens de programação e quais foram devido aos autores não terem especificado.

Dentre as pesquisas apresentadas, $71,43 \%$ informaram ter construído e aplicado um sistema específico para o tratamento dos dados dentro do AVA Moodle, sendo eles:

- inMapMoodle,

- PROTéGé e API,

- DotNetRdf,

- GVwise e

- LMS Monitor tool.

Outros informaram a utilização de coeficiente de correlação de Pearson (FILHO; JÚNIOR, 2009) por meio de técnicas de agrupamento.

Com relação aos tipos de ambientes virtuais utilizados, a análise dos dados apresentou um panorama esperado pelos autores. Esse cenário explica-se pelo fato da plataforma Moodle ter sido mais utilizada (5 artigos de 7 ), com relação a outras plataformas disponíveis, essa plataforma pode ser considerada como a mais empregada tanto no meio acadêmico como profissional. Entre os artigos selecionados 71,43\% informaram que utilizaram a plataforma Moodle, $14,29 \%$ relatou que utilizava AVA sem identificá-lo e 14,29\% informou que utilizou Ambientes Virtuais (AV), dentre esses podem ser consideras as Redes Sociais (RS).

Dentro dos trabalhos selecionados, as aplicaçõ es das pesquisas foram realizadas em sua maioria em cursos $\operatorname{EAD}(71,43 \%)$, em seguida foi identificada a modalidade de Ensino Híbrido $(14,29 \%)$ e por fim cursos On line $(14,29 \%)$, esta modalidade não ficou clara dentro do trabalho.

Materiais complementares relacionados a revisão dos trabalhos correlacionados encontram-se disponibilizados para consulta no endereço do DOI: http://dx.doi.org/10.17632/sbyfzp22tr.5

\section{Conclusões}

Estamos em um momento tecnológico de grande geração de dados e aumento no intercâmbio de saberes entre os sujeitos (OLIVEIRA et al., 2017; PEDRO; RAZERA, 2018), em que as Instituições de vários níveis de Ensino, a partir da utilização de plataformas produtos - softwares didáticos, empregando métodos e ferramentas para 
coletar, medir e processar a ampla quantidade de dados produzidos pelos alunos com relação às suas açõ es realizadas ou não dentro dos ambientes virtuais, tem condiçõ es de promover melhores estruturas para atender as necessidades de uma população diversificada de alunos (ALEXANDER et al., 2019) como subsidiar os docentes que trabalham junto a esses.

Tendo em vista o cenário apresentado, esta análise teve como objetivo verificar a utilização de sistemas baseados em Learning Anlytics para apoiar as açõ es pedagó gicas em AVA, especificamente Moodle, junto aos tutores de curso na modalidade EAD. Uma primeira visão sobre os trabalhos publicados nos ú ltimos quatro anos apresentou um alto nú mero de trabalhos com foco principal no aluno e ferramentas de cunho educacional construídas especificamente para auxiliá-los na realização de suas atividades educacionais dentro dos ambientes de apoio a aprendizagem, através de envios de mensagens e indicações de materiais de apoio.

Dentro deste contexto, esta análise procurou verificar quais são as metodologias de tratamento de dados educacionais que estão sendo utilizados pelas Instituições de Ensino, tendo como foco Learning Analytics (L.A.) e demais que estejam sendo utilizadas separada ou concomitantemente. A utilização de determinadas metodologias de tratamento de dados educacionais pode refletir no formato da entrega de informaçõ es com soluçõ es que auxiliem os tutores com relação às suas atividades pedagó gicas junto aos alunos. A partir dos resultados obtidos pode-se confirmar uma grande aderência com relação a utilização de Learning Anlytics como metodologia de trabalho junto aos dados educacionais.

Ao investigar quais são os sujeitos que estão sendo o foco das pesquisas com relação a utilização das informaçõ es geradas pelos sistemas, a pesquisa mostrou que os principais sujeitos envolvidos nas pesquisas foram os Tutores $(57,14 \%)$ e Professores $(42,86 \%)$, além de apresentar um trabalho como tendo foco nos Docentes e Tutores.

Para Alexander et al. (2019), entender como usar metodologias como LA para informar o progresso do aluno pode ser difícil para os gestores e professores das instituiçõ es de ensino, pois a necessidade de distinguir entre os diferentes tipos de dados produzidos pelos alunos é uma habilidade relativamente nova.

Outro ponto verificado nesta análise foi a construção de sistemas específicos para o tratamento dos dados educacionais pelas Instituiçõ es de Ensino, como também, a diversidade de linguagens de programação utilizadas para o desenvolvimento dos referidos sistemas.

Em relação as modalidades de curso que estão utilizando sistemas de análise de dados educacionais baseados em $L A$, além dos curso de modalidade $\operatorname{EAD}(71,43 \%)$ em acordo com o esperado, também apresentaram a utilização de $L E$ curso no formato de Ensino Híbrido e On line (14,29\%).

Com base nos achados, esta análise sugere novas pesquisas nesta área sejam realizadas para verificar a utilização dos sistemas apontados e verificação de novas implementaçõ es de metodologias e sistemas para subsidiar e apoiar as açõ es dos docentes que atuam em ambientes virtuais.

\section{Referências}

ALEXANDER, B. et al. EDUCAUSE Horizon Report 2019 Higher Education Edition. 
[S.I.], 2019.

FILHO, D. B. F.; JÚNIOR, J. A. d. S. Desvendando os mistérios do coeficiente de correlação de pearson (r). Universidade Federal de Pernambuco, 2009.

NUNES, V. B. O papel do tutor na educação a distância: como tem sido concebido pelas instituições de ensino. In: CONGRESSO INTERNACIONAL ABED DE EDUCAÇÃOA DISTÂNCIA. [S.I.: s.n.], 2013. v. 19.

OLIVEIRA, A. T. E. de et al. Ferramentas e estratégias de interação e comunicação na prática da tutoria em ead. Revista Evidência, v. 13, n. 13, 2017.

PEDRO, N.; RAZERA, F. A influência do papel do tutor na interação em fóruns de discussão: um estudo em ead com base na análise de redes sociais. Revista e-Curriculum, v. 16, n. 2, p. 467-493, 2018.

ZIELINSKI, F. D. C.; SCHMITT, M. A. R. Uma ferramenta gráfica para suporte à atividade docente no Moodle. Renote, CINTED/UFRGS, v. 13, n. 1, p. 1-10, 82015. Disponível em: (http://seer.ufrgs.br/index.php/renote/article/view/57644). 